OPEN ACCESS

Edited by:

Renzhi Wang

Peking Union Medical College Hospital (CAMS), China

Reviewed by: Vera Chesnokova, Cedars Sinai Medical Center, United States

José Miguel Hinojosa-Amaya, Autonomous University of Nuevo León, Mexico

${ }^{*}$ Correspondence: Zengyi Ma zengyima@foxmail.com Xiang Zhou xiangzhou98@outlook.com

${ }^{\dagger}$ These authors have contributed equally to this work and share first authorship

Specialty section: This article was submitted to Pituitary Endocrinology, a section of the journal

Frontiers in Endocrinology

Received: 02 September 2020 Accepted: 01 December 2020

Published: 18 January 2021

Citation:

Shen Y, Ji C, Jian X, Zhou J, Zhang Q, Qiao N, Zhang $Y$, Shou $X$, Zhou $X$ and Ma $Z$ (2021) Regulation of the EGFR Pathway by HSP9O Is Involved in the Pathogenesis of Cushing's Disease.

Front. Endocrinol. 11:601984. doi: 10.3389/fendo.2020.601984

\section{Regulation of the EGFR Pathway by HSP90 Is Involved in the Pathogenesis of Cushing's Disease}

\author{
Yue Shen ${ }^{1,2 \dagger}$, Chenxing Ji ${ }^{1,2 \dagger}$, Xuemin Jian ${ }^{3}$, Juan Zhou ${ }^{3}$, Qilin Zhang ${ }^{1,2}$, Nidan Qiao ${ }^{1,2}$, \\ Yichao Zhang ${ }^{1,2}$, Xuefei Shou ${ }^{1,2}$, Xiang Zhou ${ }^{1,2^{*}}$ and Zengyi Ma ${ }^{1,2^{*}}$ \\ ${ }^{1}$ Department of Neurosurgery, Huashan Hospital, Shanghai Medical College, Fudan University, Neurosurgical Institute of \\ Fudan University, Shanghai Clinical Medical Center of Neurosurgery, Shanghai Key Laboratory of Brain Function Restoration \\ and Neural Regeneration, Shanghai, China, ${ }^{2}$ Shanghai Pituitary Tumor Center, Shanghai, China, ${ }^{3}$ Shanghai Jiao Tong \\ University School of Medicine, Bio-X Institutes, Key Laboratory for the Genetics of Developmental and Neuropsychiatric \\ Disorders (Ministry of Education), and the Collaborative Innovation Center for Brain Science, Shanghai Jiao Tong University, \\ Shanghai, China
}

Purpose: To investigate the role of heat-shock protein Hsp90 in adrenocorticotropic hormone (ACTH)-secreting cells, and to explore the potential clinical application of an inhibitor of Hsp90, 17-N-allylamino-17-demethoxygeldanamycin(17-AAG) in corticotropinomas [also known as "Cushing's disease" (CD)].

Methods: Culture of mouse pituitary tumor [AtT-20/D16v-F2 (ATCC ${ }^{\circledR}$ CRL-1795 ${ }^{\mathrm{TM}}$ )] cells and human pituitary $\mathrm{ACTH}$-secreting tumor cells were employed. Hepatocellular carcinoma cell line (HLE) was used to evaluate EGFR inhibition by 17-AAG. Cell viability was evaluated using a commercial kit. The ACTH level was measured by a radioimmunoassay. Reverse transcription quantitative polymerase chain reaction (RTqPCR) was used to measure expression of proopiomelanocortin (POMC) mRNA. Western blotting was done to measure protein levels.

Results: 17-AAG suppressed the viability and proliferation, and promoted the apoptosis, of AtT-20/D16v-F2 cells. 17-AAG suppressed the synthesis and secretion of ACTH in AtT-20/D16v-F2 cells and down-regulated POMC transcription. 17-AAG acted in a similar pattern upon treatment with human pituitary ACTH-secreting tumor cells. Inhibition by 17AAG was stronger in human pituitary ACTH-secreting tumor cells carrying the ubiquitinspecific protease-8 (USP8) mutant in comparison with cells carrying wild-type USP8.

Conclusions: The HSP90 inhibitor 17-AAG reduced the viability and secretory function of human pituitary ACTH-secreting tumor cells, and tumor cells carrying the USP8 mutant were more sensitive to 17-AAG than tumor cells carrying wild-type USP8. 17-AAG could be a potential treatment option for CD.

Keywords: epidermal growth factor receptor, Cushing's disease, Hsp90, 17- $\mathrm{N}$-allylamino-17-demethoxygeldanamycin/ tanespimycin, pathogenesis 


\section{INTRODUCTION}

Pituitary adenoma is one of the most common tumors appearing at sella. Corticotropinoma accounts for about $2 \%-6 \%$ of pituitary adenomas (1). Corticotropinoma (Cushing disease) is responsible for $\sim 85 \%$ of cases of Cushing's syndrome (2). The clinical manifestations of hypercortisolemia are weight gain, fat redistribution, central obesity, purple striae, ecchymoses, muscle atrophy, and related complications [e.g., hypertension, diabetes mellitus, hyperlipidemia, mental/psychological disorders, osteoporosis, deep-vein thrombosis (2)].

First-line treatment for $\mathrm{CD}$ is transsphenoidal surgery, which carries a remission rate of $80 \%-90 \%$ and recurrence of approximately $10-20 \%$ (3). Patients with persistent disease can undergo secondary procedure, which carries a remission rate of $\leq 50 \%$ (4). Radiotherapy or medication are options for patients who cannot achieve remission by surgery (5).

Pharmacologic therapy includes drugs that target pituitary tumors, such as dopamine-receptor agonists [e.g., cabergoline (6), octreotide (7)], the adrenal gland [e.g., ketoconazole, metoprolol, metyrapone $(5,8,9)]$, or cortisol receptors in peripheral tissues [e.g., mifepristone (10)]. Patients who do not respond to such treatment can undergo bilateral adrenalectomy, which results in permanent adrenal insufficiency (3).

In 2015, we revealed a highly frequent somatic mutation of USP8 (which encodes ubiquitin-specific protease-8); nearly $60 \%$ of CD patients carried this gain-of-function mutation (11). This mutant interfered with the interaction between USP8 and the 143-3 motif, resulting in the reduction of the degradation of epidermal growth factor receptors (EGFRs). Therefore, ACTHsecreting adenomas with a USP8 mutation displayed higher expression of the EGFR, and increased mRNA transcription of proopiomelanocortin (POMC), which is the precursor of ACTH $(11,12)$. ACTH-secreting adenomas carrying the USP8 mutation were significantly smaller than genome-wild adenomas, which indicated greater secretion of ACTH (11). Research on primary tumor cells demonstrated that knockdown of USP8 expression or EGFR blockade could inhibit ACTH secretion effectively. Inhibition of expression of USP8 or EGFR could be a potential therapeutic target for patients with corticotropinomas carrying the USP8 mutation $(11,13)$.

Heat-shock protein Hsp90 is an important "molecular chaperone" that serves as a key regulator of protein function in eukaryotic cells during stress. Hundreds of "client" proteins interfere with HSP90 and regulate conformational changes in Hsp90 during DNA repair, the immune response, and neurodegenerative changes. Because HSP90 plays an important part in several intracellular processes, it has become a potential target for treatment of various tumor types, as well as neurodegenerative diseases and autoimmune diseases (14).

17-N-allylamino-17-demethoxygeldanamycin (17-AAG) is a derivative of the antibiotic geldanamycin. 17-AAG is an HSP inhibitor, and binds to the N-terminus of HSP90; 17-AAG is being studied as an anti-tumor antibiotic (15). Several studies have shown that HSP90 inhibitors display certain therapeutic effects on immunogenic tumors (e.g., lymphomas, melanomas) and some solid tumors [e.g., liver cancer, breast cancer, non- small-cell lung cancer $(16,17)]$. Studies have also suggested that inhibition of HSP90 expression can down-regulate expression of EGFR mutants (18-20) and become potential second-line treatment for non-small-cell lung cancers (21-23).

Based on the previous research of high-frequency mutations USP8 in corticotropinoma, this study tended to explore the regulation of the EGFR pathway by HSP90 involved in the pathogenesis of the tumor, discover potential treatment for Cushing's disease, and provide evidence for precise treatment.

\section{METHODS}

\section{Cell Culture}

Mouse pituitary tumor [AtT-20/D16v-F2 (ATCC ${ }^{\circledR}$ CRL$\left.1795^{\mathrm{TM}}\right)$ ] cells (American Type Culture Collection Manassas, VA, USA) were cultured in $75 \mathrm{~cm}^{2}$ flasks with Dulbecco's modified Eagle's medium (DMEM; Gibco, Billings, MT, USA), $10 \%$ fetal bovine serum (Gibco), and $100 \mathrm{U} / \mathrm{ml}$ of penicillinstreptomycin (Invitrogen, Carlsbad, CA, USA) in an atmosphere of $5 \% \mathrm{CO}_{2} / 95 \%$ air at $37^{\circ} \mathrm{C}$. AtT-20/D16v-F2 cells were incubated with 17-AAG(Cat. No. HY-10211 MedChenExpress Co.) for $72 \mathrm{~h}$ before cell assays.

HLE cell line, adherent growing, were cultured in $75 \mathrm{~cm}^{2}$ flasks with Dulbecco's modified Eagle's medium (DMEM; Gibco, Billings, MT, USA), $10 \%$ fetal bovine serum (Gibco), and $100 \mathrm{U} / \mathrm{ml}$ of penicillin-streptomycin (Invitrogen, Carlsbad, CA, USA) in an atmosphere of $5 \% \mathrm{CO}_{2} / 95 \%$ air at $37^{\circ} \mathrm{C}$.

Freshly resected human pituitary ACTH-secreting tumors from the Department of Neurosurgery at Huashan Hospital (Shanghai Pituitary Tumor Center, Shanghai, China) were acquired and transferred to $0.5 \%$ fetal bovine serum-containing DMEM. Tumor tissue was minced into pieces $(1-2 \mathrm{~mm})$ and digested with DMEM containing $0.3 \%$ collagenase (SigmaAldrich, Saint Louis, MD, USA) and $0.15 \%$ hyaluronidase for $60 \mathrm{~min}$ at $37^{\circ} \mathrm{C}$. The mixture was filtered with a cell strainer to remove undigested tissues and centrifuged at $180 \mathrm{~g}$ for $5 \mathrm{~min}$ at room temperature. The cell pellet was resuspended in fresh growth medium. Primary tumor cells were incubated with 17 AAG for $48 \mathrm{~h}$ before analyses. Clinical manifestations of the patients were showed in Table $\mathbf{1}$.

\section{Colony-Formation Assays}

Colony-formation assays were based on clone proliferation. Briefly, AtT-20/D16v-F2 cells were seeded in 6-well plates at low density $(\sim 1000$ cells per well) The plates were treated $1 \mu \mathrm{M}$ 17-AAG and DMSO as control, and cultured for 7 days. Then, the plates were washed with phosphate-buffered saline and stained with Crystal Violet. The images of each well were scanned, and individual clones identified. The number of clones that re-generated (each colony $>50$ cells) was counted to determine colony formation.

\section{Cell-Viability Assay}

AtT-20/D16v-F2 cells were seeded in 96-well plates (Nunc, Roskilde, Denmark) at $10^{4} \mathrm{cell} / \mathrm{ml}$, and treated with $17-\mathrm{AAG}$ 
TABLE 1 | The clinical information on the six human pituitary tumors collected.

\begin{tabular}{|c|c|c|c|c|c|c|c|c|}
\hline Patients & Age & Gender & USP8 Mutant & mUFC $\mu g / 24 H$ & Serum ACTH pg/ml & Cortisol Circadian Rhythm & Tumor Size $\mathbf{c m}^{3}$ & Pathology \\
\hline $1 \#$ & 35 & M & + & 1293.30 & 95.1 & - & $0.4^{\star} 0.3^{\star} 0.3$ & Pituitary ACTHoma \\
\hline 2\# & 29 & $\mathrm{~F}$ & + & 298.52 & 75.1 & - & $0.4^{\star} 0.3^{\star} 0.3$ & Pituitary ACTHoma \\
\hline 3\# & 48 & $\mathrm{~F}$ & + & 748.70 & 50.4 & - & $1.0^{\star} 0.5^{\star} 0.5$ & Pituitary ACTHoma \\
\hline 4\# & 45 & $\mathrm{~F}$ & - & 341.25 & 45.7 & - & $1.0 * 1.0 * 1.0$ & Pituitary ACTHoma \\
\hline $5 \#$ & 22 & $\mathrm{~F}$ & - & 1132.5 & 25.94 & - & $1.0^{\star} 0.75^{\star} 0.75$ & Pituitary ACTHoma \\
\hline $6 \#$ & 31 & $\mathrm{~F}$ & - & 486.42 & 63.05 & - & $1.0^{\star} 0.75^{\star} 0.75$ & Pituitary ACTHoma \\
\hline
\end{tabular}

with concentration at $1,2,5$, and $10 \mu \mathrm{M}$ for $24,48,72$, and $96 \mathrm{~h}$. Cells incubated with DMSO(control) or a series of 2-fold-diluted concentrations of $17-\mathrm{AAG}$ for $72 \mathrm{~h}$ to evaluate $\mathrm{IC}_{50}$ Cell viability was measured using the CellTiter-Glo ${ }^{\circledR}$ Luminescent Cell Viability Assay kit according to manufacturer (Promega, Madison, WI, USA) instructions.

\section{Western Blotting}

Cells were lysed in RIPA lysis buffer (Sigma-Aldrich) complemented with a protease inhibitor cocktail (Roche, Basel, Switzerland) to obtain whole-cell lysates. Proteins were separated by electrophoresis on $4 \%-12 \%$ Bis-Tris gels and electroblotted onto polyvinylidene difluoride membranes using the TransBlot $^{\circledR}$ Turbo ${ }^{\text {TM }}$ Transfer System (Bio-Rad Laboratories, Hercules, CA, USA). Several primary antibodies were used: anti-caspase 3 (Cell Signaling Technology, Danvers, MA, USA), anti-cleaved caspase 3 (Cell Signaling Technology), antiEGFR (Abcam, Cambridge, UK), anti-phosphorylated extracellular signal-regulated kinase (pERK)1/2 (Cell Signaling Technology) and anti-ERK1/2 (Cell Signaling Technology).

\section{Reverse Transcription Quantitative Polymerase Chain Reaction}

To determine mRNA expression of POMC, RT-qPCR was carried out using SYBR Premix Ex Taq (Tli RNaseH Plus) (Takara Biotechnology, Shiga, Japan). Hypoxanthine-guanine phosphoribosyltransferase (HPRT) was used as an internal control to normalize expression. The following primers (forward and reverse, respectively) were used: 5'-ACCACGGA AAGCAACCTG-3' and 5'-GTGGCCCATGACGTACTTC-3' for POMC; 5'-ACCAGTCAACAGGGGACATAAA-3' and 5'CTGACCAAGGAAAGCAAAGTCT-3' for HPRT.

\section{Sanger Sequencing}

Detection of gene mutations was completed by Sanger sequencing。 The oligopeptide primers were designed by the Primer3 program. The primers were: Forward: 5'ATTCACCCACCAACACTGTTCATA-3', Reverse: 5'TTGTTTTCCCGATTAACTGTTGGA-3'. Dual 384-well GeneAmp PCR System9700 (Applied Biosystems) was used. DNA template 20ng, and $2^{*}$ Taq PCR Master Mix (Lifefeng Biotech) were added to each system to complete PCR amplification. Sequencing was tested by Big Dye Terminator v.3.1 kit (Applied Biosystems), and analyzed by Chromas Software (Technelysium).

\section{Radioimmunoassay}

We adopted RIA to measure ACTH secreted by primary corticotropinoma tumor cells. Before plasma ACTH measurement, we cultured primary tumor cells in a 6-well plate with a normalized cell count of $10^{\wedge} 6$ per well. The plasma concentration of ACTH was measured by a sensitive and specific commercial radioimmunoassay, Immulite One (Siemens Medical Solutions, Malvern, PA) The sensitivity of the radioimmunoassay was $4.13 \mathrm{nmol} / \mathrm{L}$ for $\mathrm{ACTH}$, and the inter- and intra-assay coefficient of variation was $5 \%-7 \%$ and $6 \%-7.9 \%$, respectively. The final results are expressed as pictograms per milliliter.

\section{ELISA}

We measured in-vitro ACTH from AtT20/D16v-F2 cell line by ELISA. We cultured AtT20/D16v-F2 cells in a 96-well palte with a normalized cell count of 5000 per well, 3 templates for each sample. We used a commercial kit (ALPCO, ACTH ELISA) in this experiment. First configure different concentrations of standards for testing to draw a standard curve. After adding sample to the provided enzyme-labeled well plate has been coated with streptavidin, add biotin-labeled antibody to each well, and then add the enzyme-labeled antibody. Allow $4 \mathrm{~h}+-30$ min reaction time at room temperature, wash the plate 5 times, then add tetramethylbenzidine (color developer), and check the result with a spectrophotometer.

\section{Statistical Analyses}

Statistical analyses were undertaken using SPSS v25.0 (IBM, Armonk, NY, USA). Prism 7.0 (GraphPad, San Diego, CA, USA) was used to prepare graphs. The Student's t-test was used for continuous variables. The Pearson chi-square test was employed for categorical variables. $\mathrm{P}<0.05$ was considered significant.

\section{RESULTS}

\section{7-AAG Inhibits the Viability of AtT-20/ D16v-F2 Cells}

First, we demonstrated that 17-AAG could inhibit cell survival for a long time using colony-formation assays. No colony was formed in the petri dish treated with 17-AAG, whereas the mock petri dish presented with $77.8 \%$ colony formation (Figure 1). 

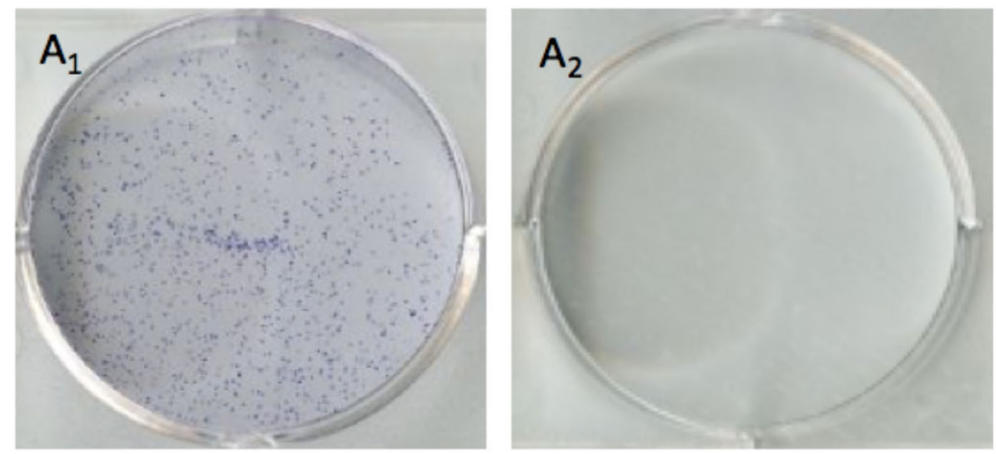

B

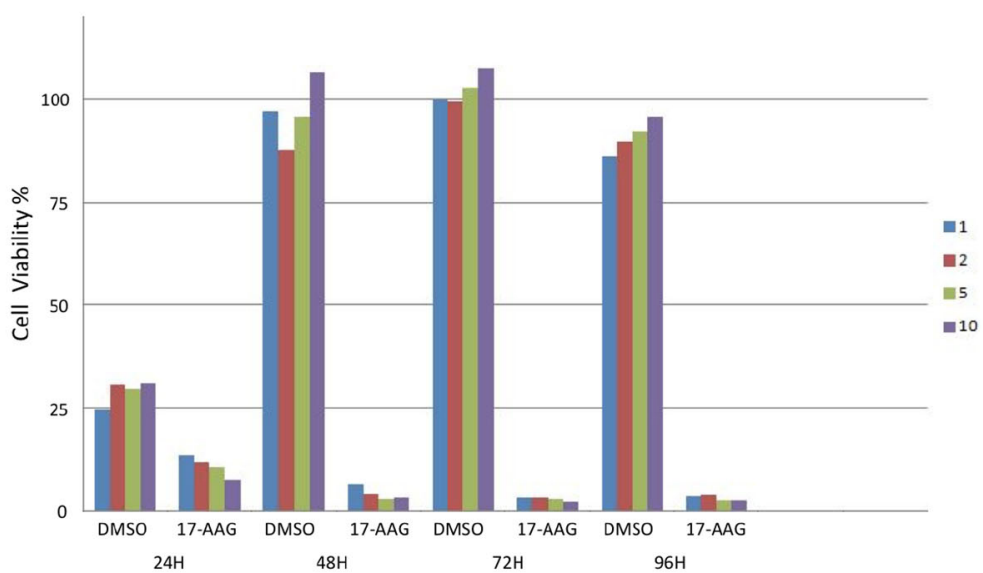

C

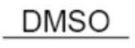

17-AAG

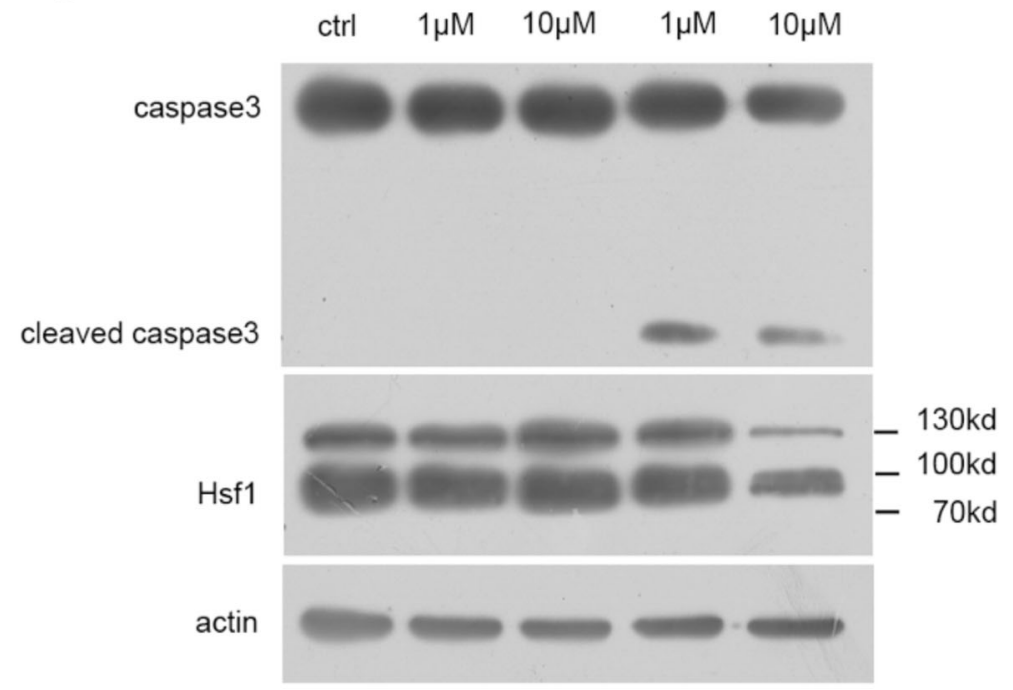

FIGURE 1 | 17-AAG inhibits the viability of AtT-20/D16v-F2 cells. In the latter, colony formation was suppressed by 17-AAG (1 $\mu$ M) (A). Caspase-3 expression demonstrated that 17-AAG promoted apoptosis of AtT-20/D16v-F2 cells (C). Proliferation of AtT-20/D16v-F2 cells was suppressed by 17-AAG remarkably in a dose- and time-dependent manner (B). All the experiments were repeated three times with consistent results.

Caspase-3 expression measured by immunoblotting showed 17-AAG promoted apoptosis of AtT-20/D16v-F2 cells (Figure 1C). Caspase-3 expression was decreased in
17-AAG-treated AtT-20/D16v-F2 cells, whereas expression of cleaved caspase- 3 was increased, suggesting that 17-AAG activated programmed cell death. Hence, 17-AAG not only 
inhibited proliferation of AtT-20/D16v-F2 cells, it also promoted their apoptosis.

We treated AtT-20/D16v-F2 cells with 17-AAG (1, 2, 5, and 10 $\mu \mathrm{M})$ for 24, 48, 72, and $96 \mathrm{~h}$ (Figure1B). Viability of AtT-20/D16vF2 cells was suppressed according to drug dose and exposure time. Upon treatment for $>72 \mathrm{~h}$, proliferation stopped, so we chose $72 \mathrm{~h}$ as the treatment time to measure the half-maximal inhibitory concentration $\left(\mathrm{IC}_{50}\right)$.

\section{7-AAG Suppressed of Proliferation of AtT-20/D16v-F2 Cells and ACTH Secretion} proliferation of AtT-20/D16v-F2 cells was found to be effectively inhibited by 17-AAG in a dose-dependent manner (Figure 2A). AtT-20/D16v-F2 cells were treated for $72 \mathrm{~h}$ with a maximum dose of $20 \mu \mathrm{M}$ of 17-AAG, and their viability was assessed. The $\mathrm{IC}_{50}$ was calculated to be $2.33 \mu \mathrm{M}$.

AtT-20/D16v-F2 cells were treated with different concentrations of 17-AGG. Cell supernatants were collected for ACTH level measurement(Figure 2B). ACTH secretion from AtT-20/D16v-F2 cells was restrained after $17-\mathrm{AAG}$ treatment, and the inhibitory effect of 17-AAG was dose-dependent. ACTH secretion from AtT20/D16v-F2 cells was impaired more remarkably after $48 \mathrm{~h}$ than $24 \mathrm{~h}$, and secretion was similar upon treatment with higher doses of 17-AGG. When treated with a high dosage of $10 \mu \mathrm{M}$ for $48 \mathrm{~h}$, ACTH level would reduce approximately $40 \%$ from baseline.

Further experiments were conducted to investigate the effect of 17-AAG on expression of POMC mRNA in AtT-20/D16v-F2 cells (Figure 2C). The latter were treated with different doses of 17AAG for 24 and $48 \mathrm{~h}$, respectively, and mRNA expression of POMC was determined by RT-qPCR. 17-AAG restrained transcription of POMC in AtT-20/D16v-F2 cells in a dose-related manner. POMC transcription level reduced approximately $75 \%$ from baseline when treated with a high dosage of $10 \mu \mathrm{M}$ for $48 \mathrm{~h}$.

\section{7-AAG Inhibited Cell Viability and Secretion of Human Pituitary ACTH-Secreting Tumor Cells}

After $48 \mathrm{~h}$ exposure to $17-\mathrm{AAG}$ at dosage of $0.5,2$, and $10 \mu \mathrm{M}$, we measured the ACTH concentration in cell supernatants (Figure 3A). We found reduced levels of ACTH secretion from primary human ACTH-secreting pituitary tumor cells in a dose-related manner compared with that in the blank control group. In USP8 mutant group, the ACTH level was suppressed to about 39\% from baseline, while in wild type group just to $82 \%$ when primary cells treated with $10 \mu \mathrm{M}$. Therefore, we examined the mechanism by which 17-AAG may affect ACTH secretion. Cell-viability tests showed that decreased secretion of ACTH might be related to the reduced number of living cells (Figure 3B), which suggested that the antitumor effect of 17-AAG partially through inhibition of cytostatic activity. In USP8 mutant group, the cell viability was suppressed to about $54 \%$ from baseline, while in wild type group just to $84 \%$ when primary cells treated with $10 \mu \mathrm{M}$. Furthermore, qRT-PCR detected reduced transcription of POMC by 17-AAG treatment. (Figure 3C). In the USP8 mutant group, 17-AAG inhibited POMC translation significantly (74\% inhibition, $\mathrm{p}=0.02$ ); While a trend towards reduced POMC expression was noted in the wild type group (63\%inhibition, $\mathrm{p}=0.06$ ), which meant the therapeutic validity manifested on function damage in tumor cells carrying the USP8 mutant were more sensitive.

\section{EGFR and Its Downstream Pathway Is Suppressed by 17-AAG in a Model Cell Line}

AtT-20/D16v-F2 cells showed negative EGFR expression (Figure 4A), so we used the HLE instead. 17-AAG $(0.2 \mu \mathrm{M})$ could inhibit EGFR expression in our model cell line. Therefore, we continue to increase the drug concentration. We found that the EGFR expression in model cells decreased after 17-AAG treatment, as well as HSP90. We further used EGF to activated cells, and discovered that the pERK1/2 was lower in the 17-AAG treated group compared to the blank control. This indicated that HSP90 inhibition arrested the downstream mitogen-activated protein kinase (MAPK) pathway, which was activated to generate ACTH (Figure 4B). From Figure 4C, we found that at the dosage of $0.5 \mathrm{nM}$ and $1.0 \mathrm{nM}, 17-\mathrm{AAG}$ could limit the inhibition of EGFR expression significantly. After autophosphorylation, EGFR bound directly or indirectly to Grb-2 to activate Ras, then activated the downstream silk/threonine protein kinase Raf, thereby phosphorylating ERK1/2. Deficiency of EGFR expression led to shortage of downstream p-ERK1/2. Therefore, we treated HLE cells with 17-AAG and EGF for further experiment (Figures 4B, C). We found that the EGFR expression in model cells decreased after 17-AAG treatment, as well as HSP90. We further used EGF to activated cells, and discovered that the p-ERK1/2 was lower in the 17-AAG treated group compared to the blank control. This indicated that HSP90 inhibition arrested the downstream mitogen-activated protein kinase (MAPK) pathway, which was activated to generate ACTH. At the same time we found, even treated with high concentration of 17-AAG, the restrain to USP8 expression were still slightly. Thus, HSP90 inhibitors were not capable to intervene USP8 expression.

\section{DISCUSSION}

Unlike the dopamine-receptor agonists acting sensitively on prolactinomas, or somatostatin analogs acting on growth hormone-secreting adenomas, pharmacologic options for ACTHsecreting adenomas are limited to drugs targeting pituitary tumors, adrenal glands, or peripheral glucocorticoid receptors. Ketoconazole can inhibit cortisol synthesis, leading to a normal level of cortisol in $50 \%$ of patients, but its use is accompanied with hepatotoxicity.

Our research team had revealed a high prevalence $(\sim 60 \%)$ of USP8 mutations in ACTH-secreting adenomas (11). This mutation exists only in corticotropinomas, suggesting that USP8 may play an important part in CD pathogenesis. Immunohistochemical studies have confirmed strong overexpression of HSP90 in AtT-20/D16vF2 cells, as well as the ACTH-secreting tumor tissue (24). 17-AAG inhibit HSP90 function to induce anti-tumor activity.

Studies have revealed the mechanism of USP8-related secretion in ACTH-secreting adenomas $(11,12)$. Different from genome-wild USP8 Deubiquitinating enzymes(DUBS), USP8 mutants cannot be phosphorylated or bind to 14-3-3 protein to activate USP8. 
A

AtT20 cell line treated by 17-AAG

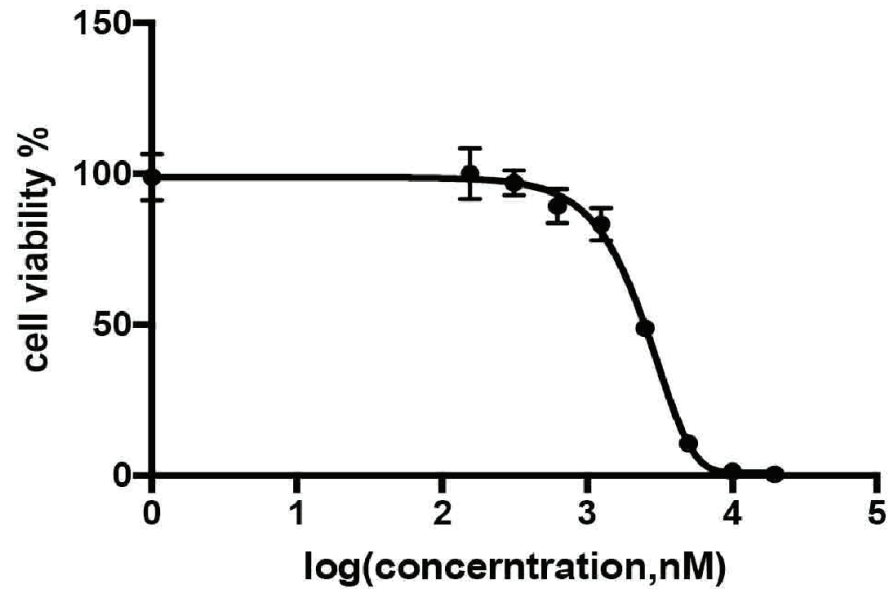

B

AtT20 cell line treated by 17-AAG

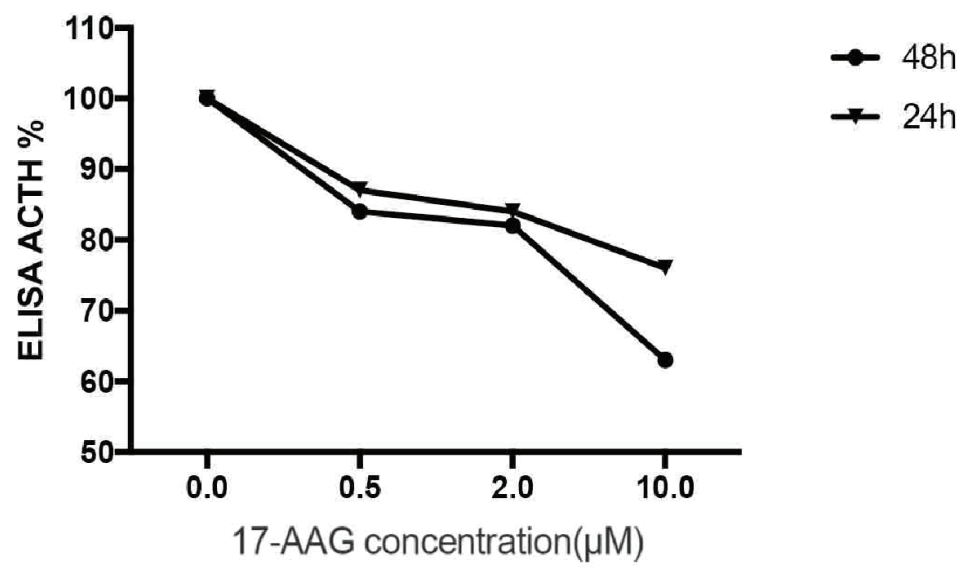

C

AtT20 cell line treated by 17-AAG

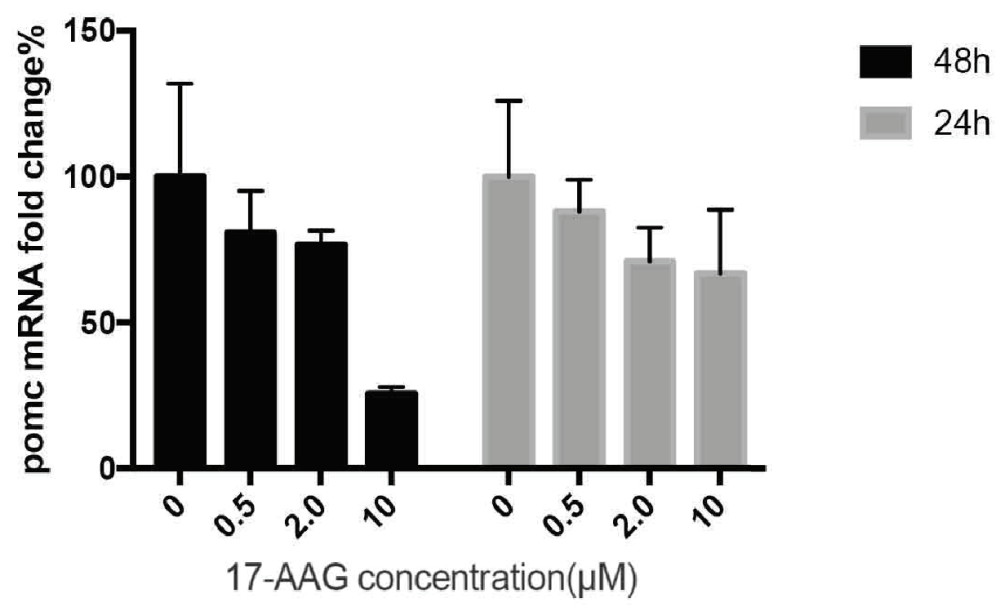

FIGURE 2 | 17-AAG suppressed proliferation of AtT-20/D16v-F2 cells and ACTH secretion. The IC 50 of 17-AAG on AtT-20/D16v-F2 cells was restrained significantly after 17-AAG treatment in a dose-related manner (B). 17-AAG restrained transcription of POMC in AtT-20/D16v-F2 cells and exhibited dose-related effects (C). Three independent experiments were repeated for each result and three replicates were studied in each single experiment. 


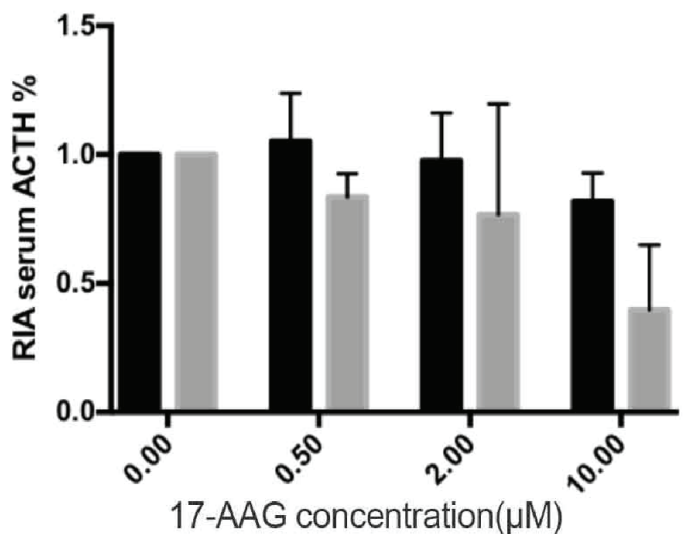

B

C

hCtT treated by 17-AAG for $48 \mathrm{~h}$

hCtT treated by $17-A A G$ for $48 \mathrm{~h}$

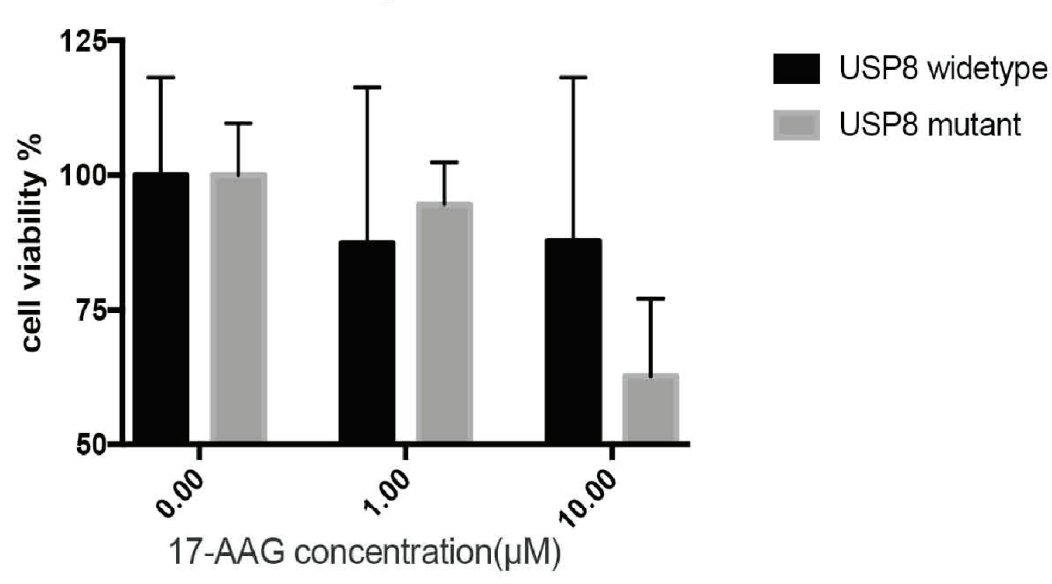

USP8 widetype

USP8 mutant

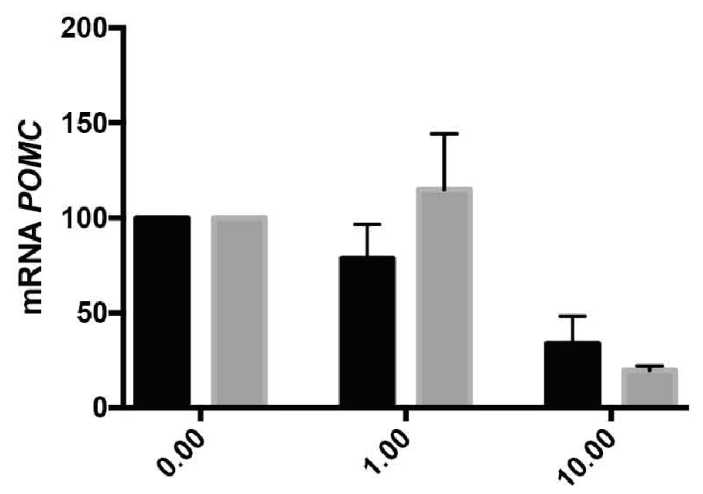

USP8 widetype

USP8 mutant

17-AAG concentration $(\mu \mathrm{M})$

FIGURE 3 | 17-AAG inhibited proliferationof primary ACTH-secreting tumor cells and ACTH secretion. Such inhibition was more remarkable in tumor cells carrying the USP8 mutant. Reduced secretion of ACTH from primary ACTH-secreting tumor cells occurred in a dose-related manner compared with that in the blank control group (A). The viability (B) and POMC transcription (C) were reduced when primary ACTH-secreting tumor cells were treated with 17-AAG, especially in the USP8mutant group. Three independent experiments were repeated for each result and three replicates were studied in each single experiment. 


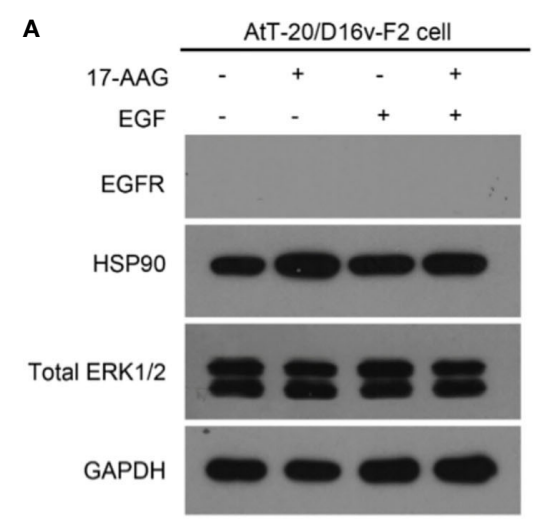

B

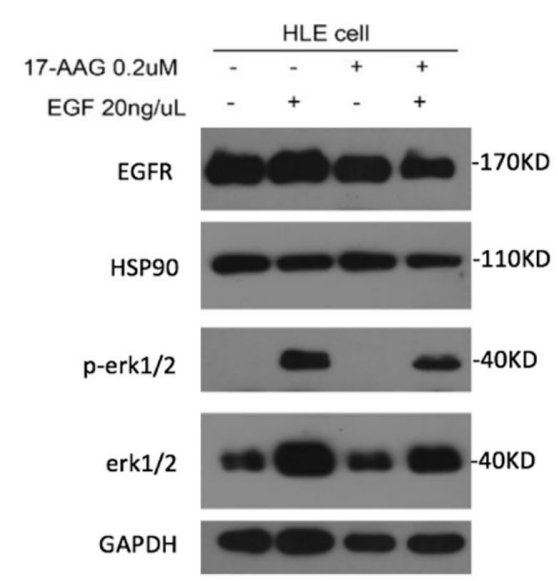

C

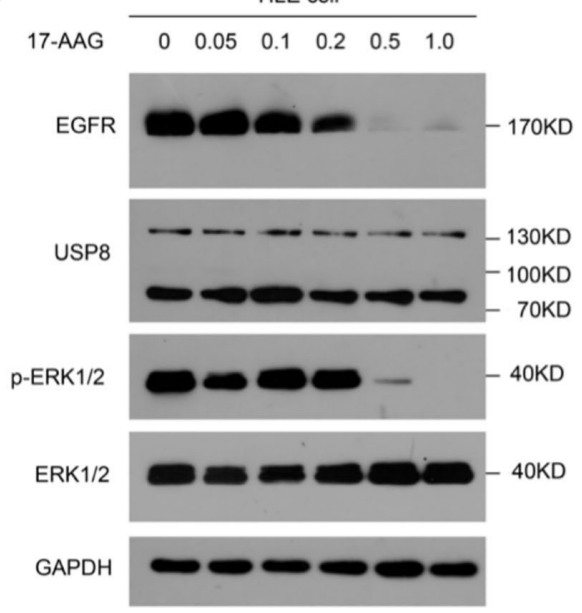

FIGURE 4 | EGFR and its downstream pathway was suppressed by 17-AAG in HLE cells.AtT-20/D16v-F2 cells did not have EGFRS (A), so we used HLE cells instead. Western blotting demonstrated expression of EFGRs and p-ERK1/2 to be down-regulated by 17-AAG treatment (B, C), which suggested that EGFR and its downstream MAPK pathway were suppressed by the HSP90 inhibitor 17-AAG. All the experiments were repeated three times with consistent results.

The function of USP8 is to deubiquitinate molecules and prevent client proteins from degradation. Accumulation of the EGFR causes up-regulation of signaling pathways and thereby accelerates POMC transcription by inducing the deregulation of p27 $(25,26)$, or degradation of other regulators such as histone deacetylase. Hence, inhibition of USP8 and/or EGFRs represents a potential remedy for CD. Studies in primary tumor cells with USP8 mutants have shown that gefitinib treatment (EGFR inhibitor) can reduce ACTH secretion $(11,13)$.

When 17-AAG acted upon AtT-20/D16v-F2 cells, it suppressed their colony formation and viability remarkably. Cell proliferation was constrained considerably, with an $\mathrm{IC}_{50}$ of $2.33 \mu \mathrm{M}$. Connections between apoptosis and ACTH and 17-AAG were found by measurement of caspase- 3 expression. Further analyses showed the positive correlation between the 17-AAG concentration and ACTH secretion in AtT-20/D16v-F2 cells was based on the drug dose and exposure time. RT-qPCR also confirmed the conclusion with POMC transcription result. Experiments on AtT-20/D16v-F2 cells suggested that $17-\mathrm{AAG}$ could suppress their viability and ACTH secretion.
Because of the deficiency of EGFR expression in AtT-20/D16vF2 cells and difficulty in obtaining human corticotropinomas cell line, we used a model cell line HLE which delivered EGFR and human primary tumor cells. In primary ACTH-secreting tumor cells we verified those findings and, interestingly, mutant tumor cells exhibited more sensitivity towards 17-AAG in terms of cell viability and POMC transcription. Expression of EGFRs and p-ERK1/2, which activated in the downstream MAPK pathway, was reduced distinctly by $17-\mathrm{AAG}$ within dosage-related manner. Geldanamycin(GA), a specific inhibitors of the cytosolic chaperone HSP 90, were shown to accelerate degradation of the EGFR (27). GA blocks processing of newly synthesized EGFR. The effects of GA on receptor degradation are mediated by the cytosolic portion of EGFR and could be conferred to the erythropoietin receptor (EPO-R), by employing the respective chimera. But GA didn't affect stability of newly synthesized EGFR lacking the cytosolic domain (Ex EGFR) (27).However, Geldanamycin has not been used in clinical scenario because of liver toxicity. 17-AAG is a new derivative of geldanamycin that 
shares its important biological activities but shows less toxicity (28). Hence, EGFR function could be restrained and POMC transcription mediated by the MAPK pathway reduced after inhibition of HSP90 expression. These actions would contribute to tumor-growth inhibition in signaling pathways involving ACTH secretion.

While, in the context of USP8 mutated, the deregulation of EGFR led to improve MAPK signaling and subsequently promoted POMC transcription, which further result to the oversecretion of ACTH. Invitro model cell experiments also confirmed that HSP90 inhibitor could significantly reduce the expression level of EGFR and lower down-regulate the MAPK pathway. We believed that overexpressed EGFR provided greater targets for HSP90 inhibitors, therefore 17AAG manifested a more significant effect on USP8 mutant tumor cells.

HSP90, as a molecular chaperone, plays a key role in the conformational maturation of oncogenic signaling proteins. HSP90 inhibitors selectively kill cancer cells compared to normal cells, and have also demonstrated antitumor effects towards lymphomas, melanomas, as well as some solid tumors (e.g., breast cancer, non-small-cell lung cancer) $(16,17)$. To date the majority of pharmaceutical research and development focused on targeting the $\mathrm{N}$-domain ATP binding site of HSP90, C-terminal of the protein on the heels. Silibinin, as well as novobiocin, were the prototypic inhibitors that interact at the C-domain site. And the therapeutic effect in cancer had been demonstrated. One conceivable benefit of these medication is that certain C-terminal inhibitors appear to have weaker HSF1 activation capacity. The ongoing study support further optimization of medicinal chemistry and preclinical evaluation of C-terminal Hsp90 inhibitors. The four Hsp90 subtypes (Hsp90 $\alpha$ and Hsp90 $\beta$ in the cytoplasm and nucleus, the relative importance of GRP94 and TRAP1 in the endoplasmic reticulum in cancer) and mitochondria were still under research (29). Our present study provides a foundation for potential clinical treatment of CD by HSP90 inhibitor. Further research will continue to build animal models to improve related in-vivo experiments. It will provide more detailed research basis for the clinical application of HSP90 inhibitors in the treatment of Cushing's disease.

\section{CONCLUSIONS}

The HSP90 inhibitor 17-AAG reduced the viability and secretory function of human pituitary ACTH-secreting tumor cells, and tumor cells carrying the USP8 mutant were more sensitive to 17-

\section{REFERENCES}

1. Clayton RN, Jones PW, Reulen RC, Stewart PM, Hassan-Smith ZK, Ntali G, et al. Mortality in patients with Cushing's disease more than 10 years after remission: a multicentre, multinational, retrospective cohort study. Lancet Diabetes Endocrinol (2016) 4(7):569-76.

2. Pivonello R, Isidori AM, De Martino MC, Newell-Price J, Biller BMK, Colao A. Complications of Cushing's syndrome: state of the art. Lancet Diabetes Endocrinol (2016) 4(7):611-29.

3. Bertagna X, Guignat L. Approach to the Cushing's disease patient with persistent/ recurrent hypercortisolism after pituitary surgery. J Clin Endocrinol Metab (2013) 98(4):1307-18.
AAG than tumor cells carrying wild-type USP8. 17-AAG could be potential clinical treatment for CD.

\section{DATA AVAILABILITY STATEMENT}

The original contributions presented in the study are included in the article/supplementary material. Further inquiries can be directed to the corresponding authors.

\section{ETHICS STATEMENT}

Written informed consent was obtained from the individual(s) for the publication of any potentially identifiable images or data included in this article.

\section{AUTHOR CONTRIBUTIONS}

Conception or design of the work: ZM and XZ. Financial support: ZM, QZ, and YZ. Surgical specimen recruitment: NQ, YZ, XS, XZ, ZM. Laboratory Practice: YS, CJ. Data analysis and interpretation: YS, ZM, QZ. Drafting the article: YS, CJ. Critical revision of the article: $\mathrm{ZM}, \mathrm{XZ}$. All authors contributed to the article and approved the submitted version.

\section{FUNDING}

The research was supported by the National Science Fund for Distinguished Young Scholars (81725011), Clinical Research Plan of SHDC(SHDC2020CR2004A) to YZ; the National Natural Science Funds of China (81702467), Shanghai Sailing program (17YF1401500) to ZM; National Natural Science Funds of China (81802495), Shanghai Sailing program (18YF1403400) to $\mathrm{QZ}$.

\section{ACKNOWLEDGMENTS}

We gratefully acknowledge Prof Yao Zhao for funding support. We thank Mrs. Yun Zhang, Mrs. Ye Wang, Mrs. Qiuyue Wu and Mrs. Lan Lai for sample collection.

4. Ram Z, Nieman LK, Cutler GB, Chrousos GP, Doppman JL, Oldfield EH. Early repeat surgery for persistent Cushing's disease. J Neurosurg (1994), 37-45, 80(1).

5. Castinetti F, Guignat L, Giraud P, Muller M, Kamenicky P, Drui D, et al. Ketoconazole in Cushing's disease: is it worth a try? J Clin Endocrinol Metab (2014) 99(5):1623-30.

6. Molitch ME. Current approaches to the pharmacological management of Cushing's disease. Mol Cell Endocrinol (2015) 408:185-9.

7. Colao A, Petersenn S, Newell-Price J, Findling JW, Gu F, Maldonado M, et al. A 12-month phase 3 study of pasireotide in Cushing's disease. New Engl J Med (2012) 366(10):914-24.

8. Preda VA, Sen J, Karavitaki N, Grossman AB. Therapy In Endocrine Disease: Etomidate in the management of hypercortisolaemia in Cushing's syndrome: a review. Eur J Endocrinol (2012) 167(2):137-43. 
9. Daniel E, Aylwin S, Mustafa O, Ball S, Munir A, Boelaert K, et al. Effectiveness of metyrapone in treating Cushing's syndrome: a retrospective multicenter study in 195 patients. J Clin Endocrinol Metab (2015) 100(11):4146-54.

10. Fleseriu M, Biller BM, Findling JW, Molitch ME, Schteingart DE, Gross C, et al. Mifepristone, a glucocorticoid receptor antagonist, produces clinical and metabolic benefits in patients with Cushing's syndrome. J Clin Endocrinol Metab (2012) 97(6):2039-49. doi: 10.1210/jc.2011-3350

11. Ma ZY, Song ZJ, Chen JH, Wang YF, Li SQ, Zhou LF, et al. Recurrent gain-offunction USP8 mutations in Cushing's disease. Cell Res (2015) 25(3):306. doi: 10.1038/cr.2015.20

12. Reincke M, Sbiera S, Hayakawa A, Theodoropoulou M, Osswald A, Beuschlein F, et al. Mutations in the deubiquitinase gene USP8 cause Cushing's disease. Nat Genet (2015) 47(1):31.

13. Fukuoka H, Cooper O, Ben-Shlomo A, Mamelak A, Ren SG, Bruyette D, et al. EGFR as a therapeutic target for human, canine, and mouse ACTH-secreting pituitary adenomas. J Clin Invest (2011) 121(12):4712-21.

14. Taldone T, Gozman A, Maharaj R, Chiosis G. Targeting Hsp90: smallmolecule inhibitors and their clinical development. Curr Opin Pharmacol (2008) 8(4):370-4.

15. Dimopoulos MA, Mitsiades CS, Anderson KC, Richardson PG. Tanespimycin as antitumor therapy. Clin Lymphoma Myeloma Leukemia (2011) 11(1):17-22.

16. Usmani SZ, Chiosis G. HSP90 inhibitors as therapy for multiple myeloma. Clin Lymphoma Myeloma Leukemia (2011) 11:S77-81.

17. J haveri K, Chandarlapaty S, Lake D, Gilewski T, Robson M, Goldfarb S, et al. A phase II open-label study of ganetespib, a novel heat shock protein 90 inhibitor for patients with metastatic breast cancer. Clin Breast Cancer (2014) 14(3):154-60.

18. Johnson ML, Yu HA, Hart EM, Weitner BB, Rademaker AW, Patel JD, et al. Phase I/II study of HSP90 inhibitor AUY922 and erlotinib for EGFR-mutant lung cancer with acquired resistance to epidermal growth factor receptor tyrosine kinase inhibitors. J Clin Oncol (2015) 33(15):1666.

19. Yang YC, Cheng TY, Huang SM, Su CY, Yang PW, Lee JM, et al. Cytosolic PKM2 stabilizes mutant EGFR protein expression through regulating HSP90EGFR association. Oncogene (2017) 36(29):4234.

20. Jorge SE, Lucena-Araujo AR, Yasuda H, Piotrowska Z, Oxnard GR, Rangachari D, et al. EGFR exon 20 insertion mutations display sensitivity to Hsp90 inhibition in preclinical models and lung adenocarcinomas. Clin Cancer Res (2018) 24(24):6548-55.

21. Wang CY, Guo ST, Wang JY, Liu F, Zhang YY, Yari H, et al. Inhibition of HSP90 by AUY922 preferentially kills mutant KRAS colon cancer cells by activating Bim through ER stress. Mol Cancer Ther (2016) 15 (3):448-59.

22. Wang CY, Guo ST, Wang JY, Yan XG, Zhang XD. Abstract 3066: inhibition of hsp90 by auy 922 preferentially kills mutant kras colon cancer cells by activating bim through er stress. Cancer Res (2017) 77(13 Supplement):3066-6.

23. Raverot G, Burman P, Mccormack A, Heaney A, Petersenn S, Popovic V, et al. European Society of Endocrinology Clinical Practice Guidelines for the management of aggressive pituitary tumours and carcinomas. Eur J Endocrinol (2018) 178(1):G1-G24.

24. Riebold M, Kozany C, Freiburger L, Sattler M, Buchfelder M, Hausch F, et al. A C-terminal HSP90 inhibitor restores glucocorticoid sensitivity and relieves a mouse allograft model of Cushing disease. Nat Med (2015) 21(3):276.

25. Lidhar K, Korbonits M, Jordan S, Khalimova Z, Kaltsas G, Lu X, et al. Low expression of the cell cycle inhibitor p27Kip1 in normal corticotroph cells, corticotroph tumors, and malignant pituitary tumors. J Clin Endocrinol Metab (1999) 84(10):3823-30.

26. Chu IM, Hengst L, Slingerland JM. The Cdk inhibitor p27 in human cancer: prognostic potential and relevance to anticancer therapy. Nat Rev Cancer (2008) 8(4):253.

27. Supino-Rosin L, Yoshimura A, Yarden Y, Elazar Z, Neumann D. Intracellular Retention and Degradation of the Epidermal Growth Factor Receptor, Two Distinct Processes Mediated by Benzoquinone Ansamycins. J Biol Chem (2000) 275(29):21850-5.

28. Kamal A, Thao L, Sensintaffar J, Zhang L, Boehm MF, Fritz LC, et al. A highaffinity conformation of Hsp90 confers tumour selectivity on Hsp90 inhibitors. Nature (2003) 425(6956):407-10.

29. Neckers L, Workman P. Hsp90 molecular chaperone inhibitors: are we there yet? Clin Cancer Res: an Off J Am Assoc Cancer Res (2012) 18(1):64-76. doi: 10.1158/1078-0432.CCR-11-1000

Conflict of Interest: The authors declare that the research was conducted in the absence of any commercial or financial relationships that could be construed as a potential conflict of interest.

Copyright ( 2021 Shen, Ji, Jian, Zhou, Zhang, Qiao, Zhang, Shou, Zhou and Ma. This is an open-access article distributed under the terms of the Creative Commons Attribution License (CC BY). The use, distribution or reproduction in other forums is permitted, provided the original author(s) and the copyright owner(s) are credited and that the original publication in this journal is cited, in accordance with accepted academic practice. No use, distribution or reproduction is permitted which does not comply with these terms. 